\title{
INTERRELACIÓN LOCAL - GLOBAL Y CIUDADANÍA PLANETARIA: UNA CUESTIÓN IMPORTANTE PARA LA EDUCACIÓN DEL SIGLO XXI
}

\author{
Olga Moreno Fernández ${ }^{1}$ \\ Dalva Maria Bianchini Bonotto ${ }^{2}$
}

Resumen: En este trabajo teórico, nuestro objetivo es aportar algunas contribuciones desde la educación ciudadana planetaria a las cuestiones relevantes de nuestro mundo y a las implicaciones sociales que genera la globalización en las prácticas ciudadanas del siglo XXI. Las problemáticas socio-ambientales y la crisis mundial en la que el planeta se encuentra inmerso precisan de respuestas urgentes acordes a las necesidades actuales. Respuestas en las que la educación tiene un papel fundamental, poniendo de manifiesto la necesidad de trabajar y entender las realidades sociales y ambientales desde una perspectiva local-global, siendo el modelo de ciudadano planetario el que mejor responde a estas necesidades.

Palabras-clave: Interrelación local-global. Educación para la ciudadanía. Educación ambiental. Ciudadanía planetaria.

\section{LOCAL - GLOBAL NETWORKING AND GLOBAL CITIZENSHIP: AN IMPORTANT ISSUE TO THE XXI CENTURY'S EDUCATION}

Abstract: In this theoretical work, we aim to contribute from planetary citizenship education to relevant issues of our world and the social implications brought by globalization in the twenty-first century citizenship practices. The socio-environmental issues and global crisis that the planet is undergoing chords require urgent responses to current needs. Responses that education has a key role, highlighting the need to work and understand the social and environmental realities from a local-global perspective, being the planetary model citizen who best answer these needs.

Keywords: Local - Global interrelation. Citizen education. Environmental education. Planetary citizenship.

\section{INTER-RELAÇÃO LOCAL-GLOBAL E CIDADANIA PLANETÁRIA: UMA QUESTÃO IMPORTANTE PARA A EDUCAÇÃO DO SÉCULO XXI}

Resumo: Neste trabalho teórico, nosso objetivo é oferecer algumas contribuições da educação para a cidadania planetária para questões relevantes do nosso mundo e as implicações sociais geradas pela globalização quanto às práticas de cidadania do século XXI. Os problemas socioambientais e a crise global que o planeta está passando exigem respostas urgentes às necessidades atuais. Respostas nas quais a educação desempenha um papel fundamental, destacando-se a necessidade de trabalhar e compreender as realidades

\footnotetext{
${ }^{1}$ Doctora en Estudios Medioambientales por la Universidad Pablo de Olavide. Docente de la Universidad de Sevilla, Sevilla, España. Facultad de Ciencias de la Educación, Departamento de Didáctica de las ciencias Experimentales y Sociales. omoreno@us.es

${ }^{2}$ Doutora em Educação pela Universidade Federal de São Carlos. Docente do Departamento de Educação Instituto de Biociências da Universidade Estadual Paulista/UNESP, Rio Claro, São Paulo, Brasil. dalvambb@rc.unesp.br
} 
sociais e ambientais de uma perspectiva local-global, sendo o cidadão planetário o modelo que melhor atende a essas necessidades.

Palavras-chave: Inter-relação local-global. Educação para a cidadania. Educação ambiental. Cidadão Planetário.

\section{1- La interrelación local y global del mundo en el que vivimos}

La promesa de un mundo de seguridad y previsibilidad, tejida a finales del siglo XIX, fue sucedida, en el siglo XX, por la realidad de un mundo incierto y complejo. Llegamos, así, al siglo XXI, a un mundo entendido como un sistema de interconexiones entre todos los componentes que lo conforman, entendiendo al planeta como un todo.

Emergiendo de este sistema, destacamos el fenómeno conocido como globalización, que, según diversos autores, entre ellos Porto-Gonçalves (2007), tuvo su inicio en momentos históricos más remotos de lo que normalmente se considera y que se puede caracterizar por el dominio del ser humano sobre el otro o sobre la naturaleza, dando como resultado grandes desigualdades. Considerando la complejidad de este fenómeno, en la actualidad se han realizado intentos de reflexionar sobre el mismo, con la finalidad de apuntar directrices de acción que enfrente sus efectos negativos.

Según Giddens (1994), mucho más allá de la dimensión económica, la globalización implica la transformación del contexto social, transformando la vida cotidiana de las personas en sus espacios locales. Con eso, lo local y lo global se encuentran intrínsecamente imbricados, de tal forma que la comprensión sobre las relaciones establecidas entre la macroescala y la microescala se ha constituido como un desafío a abordar para las ciencias de nuestra época (WILBANKS; KATES, 1999; GASKEL; OGAWA, 2004). Así como lo global influencia a lo local, podemos considerar el camino inverso, siendo esta la perspectiva sobre la cual varios autores se han apoyado.

Santos (2002), frente a la crueldad con que la presente globalización se está constituyendo, considera que es posible cambiar la forma de como esta se procesa actualmente, dejando de beneficiar apenas a los países más ricos en detrimento de los más pobres. Admitiendo que el mundo puede diferenciarse en cómo es (la globalización perversa actual) y cómo puede ser (otra globalización), ese autor destaca que las mismas bases técnicas que sirven actualmente para construir la globalización, tal cual la conocemos hoy, pueden servir para construir una globalización más humana, apuntando para la necesaria reconstrucción de las relaciones locales.

Su visión optimista del futuro implica la construcción de relaciones humanas basadas en la solidaridad. Así que se propone una revisión de la globalización, sin descartar la base técnica que soporta actualmente la globalización económica y financiera: creada para la circulación de capitales, la base puede servir para transmitir los valores humanos, para permitir una integración efectiva de los diferentes lazos culturales que permiten la construcción de los sucesos de solidaridad, tal y como lo define Santos (2000).

En este sentido, algunas experiencias que se han producido en todo el mundo ha llamado la atención de los estudiosos de estos temas. Una de ellas es el uso de internet, cuyo impacto se ha sentido en toda la sociedad, pudiendo promover el fortalecimiento de los movimientos contra-hegemónicos. Constituyéndose como una herramienta excelente, capaz de movilizar grupos de personas con intereses y objetivos comunes, dando lugar a verdaderos movimientos ciudadanos, por lo que consideramos que internet es un campo todavía por explorar en este sentido.

Eso es lo que ocurrió, por ejemplo, con el levantamiento de las comunidades indígenas de Chiapas en 2004. Situado en una región profundamente marginada y aislada de México, su 
lucha, que tuvo lugar desde finales del siglo pasado, ha contribuido al debate sobre las cuestiones de autonomía cultural, debilitamiento o desaparición de las fronteras tradicionales (geográficos, económicos, políticos y culturales) y posibilidades de construir comunidades locales y globales.

El uso de las nuevas tecnologías y la distribución de los textos en los que estos indígenas amplifican sus voces en su movimiento de resistencia fueron fundamentales en el proceso de mediación con las culturas hegemónicas mexicanas. Este movimiento nos trajo elementos importantes para el análisis de las "posibilidades que las nuevas tecnologías pueden aportar para el futuro en un tejido que conecte las luchas locales con los problemas mundiales" (ABDELMONEIM, 2002, p.47).

Mayer (2002), a su vez, analiza la globalización en su interrelación con la cuestión ambiental, resaltando el papel de la comunidad local. Según esta autora, ante la globalización que viene de arriba y es impuesta por el mercado estamos viendo la construcción de una globalización que viene de abajo, partiendo de una acción local. Denominándola como una ciudadanía glocal, la autora indica que esta viene a asumir no solamente la responsabilidad de controlar los problemas locales, reconociendo sus causas y denunciando sus riesgos, sino también a actuar en un ámbito transnacional, a partir de redes, asociaciones y organizaciones.

Señala los cambios que pasan a exigirse a nuestra sociedad, como demuestran los actuales tratados ambientales internacionales para el enfrentamiento de varios problemas, que nos remiten no solo a una elevación del nivel de conciencia colectiva con relación a la complejidad de relaciones entre la especie humana y el planeta, sino también la consolidación de

[...] un concepto distinto de ciudadanía y de estado, un concepto que incluya la responsabilidad de defender los derechos no sólo de los propios ciudadanos sino también los de los ciudadanos de otras naciones, y no sólo de los ciudadanos actuales, sino también de los ciudadanos de las generaciones futuras (MAYER, 2002, p. 88).

Es de destacar que, para nosotros, estos debates suponen que no existe un consenso general en cuanto a la problemática ambiental que está sufriendo nuestro planeta. En las últimas décadas ha habido una creciente preocupación por el medio ambiente, debido a que estamos asistiendo a un crecimiento gradual de los problemas de la degradación ambiental, que alcanzan, progresivamente, mayor gravedad y extensión. Sin embargo, reconocemos que este consenso termina cuando se trata de entender las causas de estos problemas y, por ende, la necesidad de pasar a la acción para abordarlos.

En este sentido, Loureiro (2000) presenta tres posturas problemáticas, típicas de una parte significativa de los grupos ecologistas, sobre el tratamiento de las cuestiones ambientales:

- la primera, lo que se ha dado por denominar naturalismo: en este contexto, los problemas ambientales se abordan en orden a-histórico, haciendo caso omiso de las relaciones sociales. La acción humana se define como antropogénica y se interpreta bajo los parámetros de las ciencias biológicas en el positivismo clásico;

- la segunda bajo tecnicismos: las soluciones técnicas, de gestión y manejo de los recursos naturales son vistos como capaces de resolver los dilemas natural, haciendo caso omiso de los aspectos políticos y económicos que contextualizan las opciones tecnológicas y sus consecuencias en la sociedad y privilegiando la supremacía de la razón;

- y la tercera corriente teórica se establece en el contexto del romanticismo ingenuo, que presenta al ser humano como agente nocivo. Esta generalización de categoría humana permite el uso ideológico de las cuestiones ambientales, haciendo que se ponga el foco del análisis en la estructura de la sociedad, poniendo la responsabilidad exclusivamente en el ser humano y en su instinto de destrucción. 
Estamos de acuerdo con este y otros autores, que señalan que los problemas ambientales son el resultado de una crisis más amplia, el resultado de "un conjunto de variables interrelacionadas, derivadas de categorías como el capitalismo, la modernidad, la industrialización, la urbanización y la tecnocracia" (LOUREIRO, 2000, p. 24). Lo que implica un reto adicional en la búsqueda de acuerdos y consensos - siempre provisionales - frente a las propuestas y acciones a realizar, que los eventos internacionales han dedicado al tema ambiental y que terminan quedando en simples recogidas de intenciones que no van más allá del papel.

Con estas consideraciones, nos dirigimos al campo educativo, reflexionando sobre como contribuye a paliar la crisis actual del medio ambiente, teniendo presente la interrelación localglobal que se da a nivel socio-ambiental. Una tarea que distintas tendencias educativas intentan llevar a cabo, entre ellas la educación ambiental y la educación para la ciudadanía. Se hace imprescindible educar al ciudadano para vivir y actuar en ese escenario complejo. Como destaca García Díaz (2006, p.11), resulta fundamental a la tarea educativa facilitar la comprensión de la interacción entre lo global y lo local, "que implica comprender la interacción social-natural y una tomada de consciencia global sobre el lugar del hombre en la naturaleza y sobre el papel que han de desempeñar los individuos y dos grupos sociales en las relaciones con el entorno y entre los propios humanos".

\section{2- Desafíos de la educación para hacer frente a la interrelación local-global}

En relación a la educación ambiental, desde 1972, con la Conferencia de las Naciones Unidas sobre el Medio Humano, en Estocolmo, se recomendó la creación de un programa internacional de Educación Ambiental (EA) como un importante paso para la actuación y control de los problemas ambientales.

Sin embargo, como consecuencia de las distintas formas de entender la cuestión ambiental y los propios fines de la educación, existe, desde entonces, una gran diversidad de propuestas, terminologías y prácticas acerca de la educación ambiental (SAUVÉ, 1996).

Para nosotros la educación ambiental debe comprenderse, ante todo, como un proceso de constitución del ser humano, proceso condicionado por las contradicciones sociales, pero que pueden contribuir para la transformación de las desigualdades y formación de sujetos autónomos (RODRIGUES, 2001) a través de una educación dirigida al ejercicio de la ciudadanía. Una ciudadanía cosmopolita necesaria para la constitución de una sociedad pluralista y democrática.

Estas consideraciones van al encuentro de las propuestas presentadas en el Tratado de Educación Ambiental para Sociedades Sostenibles y Responsabilidad Global, firmado por el Foro de las Organizaciones No Gubernamentales durante la ECO-92. Este resalta diversos aspectos considerados imprescindibles en la construcción de una sociedad sostenible, afirmando "valores y acciones que contribuyen para la transformación humana y social y para la preservación ecológica” (VIEZZER; OVALLES, 1994, p.29).

El documento apunta, también, la necesidad de pensar y actuar local y globalmente, superando el slogan pensar globalmente y actuar localmente, al considerar la imbricación de estas escalas en los temas y problemas ambientales, indicando, a partir de este enunciado, un importante desafío a la educación.

A partir de la ECO-92, todos los países signatarios de esa conferencia vienen intentando discutir, proponer y desarrollar programas y acciones en torno a prácticas de educación ambiental más críticas y participativas. Sin embargo, las investigaciones que permiten acompañar esas acciones han apuntado las dificultades para concretar tal propuesta, indicando que, actualmente, son poco efectivas. En este trabajo destacamos las dificultades relativas a la interrelación entre las escalas local y global de los problemas ambientales, necesaria para precisar una educación direccionada a la ciudadanía planetaria. 
Es preciso reconocer que la propuesta de comprender la realidad en su complejidad no es de fácil aprehensión, pues, educados a partir de un paradigma simplificador de la realidad, que nos acostumbró a separar, centrarnos en la parte, simplificar y reducir la realidad para comprenderla, nos quedamos presos a esa trampa paradigmática (GUIMARÃES, 2007) e, incluso intentando hacer diferente, nuestras comprensiones y nuestras prácticas tienden a ser limitadas. Así que las investigaciones apuntan que no se dedica una adecuada atención a los problemas planetarios, sino sigue predominando una aproximación local.

Vilarrasa (2005, p.15), en una investigación sobre el pensamiento y las prácticas de un grupo de profesores catalanes sobre el papel del medio local en la construcción del conocimiento social en clases de educación secundaria observó que el medio local era considerado en sí mismo: sólo en $24,6 \%$ del total de los ejercicios se establecían relaciones entre lugares y escalas diferentes, situación que dificulta la "comprensión de las interacciones local-global, tan importante para la educación para la ciudadanía en el mundo actual".

Por su parte, Moreno (2013), en su tesis doctoral, en que buscó identificar las potencialidades y limitaciones de los trabajos desarrollados en el ámbito de los programas educativos andaluces que fomentan la participación ciudadana, observó que el alumnado señalaba sentirse ciudadano del mundo, afirmando entender que las problemáticas socioambientales locales estaban también relacionadas con las problemáticas globales, aunque no se sentían capaces de relacionar esa dimensión glocal. Sin embargo, el profesorado asumía que a estas edades (12-16 años) el alumnado no era capaz de ver más allá de su entorno local, no dándole así la oportunidad de poder relacionarse con su mundo de una forma más compleja y completa, además de necesaria.

$\mathrm{Y}$ es que, muchos educadores ambientales enfatizan la pertinencia del trabajo inicial o principal - con la escala local, que estaría dirigida a la realidad próxima de los estudiantes, siendo más accesible y comprensible.

En una investigación que incluyó algunos profesores de la enseñanza secundaria (alumnos de 11-14 años de edad), participantes de un curso de educación ambiental en una ciudad de la región sureste brasileña (DIAS; BONOTTO, 2012), se observó la mayor frecuencia y desenvoltura con la que los profesores comentaban durante el curso sobre la importancia de la valoración del local/ cotidiano/ realidad de los alumnos. En el intento de articular las dos escalas algunos profesores intentaron establecer conexiones, que, en la mayoría de las veces, se mostraron un tanto difusas e inconsistentes. La investigación reveló la falta de conocimientos generalizada sobre esta cuestión.

Layrargues (1999), discutiendo el interés en las prácticas educativas de EA centradas en la resolución de problemas locales, apunta para el peligro de transformar tales actividades en actividades fines, llamando la atención para la pérdida de la comprensión más amplia de la problemática que los envuelve. Para el autor, los problemas ambientales no pueden reducirse a actividades fines, cuya solución se dé sea por una resolución puntual de un problema local, sea por cambios comportamentales de cada individuo. Para una propuesta crítica de EA, formadora de la ciudadanía, los problemas ambientales deben ser temas generadores que problematicen la realidad para comprenderla de una forma más profunda, instrumentalizándola para una acción crítica de sujetos en proceso de concienciación.

La falta de actividades educativas que impliquen una articulación entre los niveles local y global también se pueden constatar a partir de la investigación realizada por Dias y Bonotto (2014), que pretendía trazar y analizar la producción académica brasileña relacionadas con la educación ambiental, teniendo en cuenta el trabajo que se centraban en la escala local y global. La investigación implicó el análisis de los resúmenes de tesis y disertaciones que se encuentran en el Banco de Teses da Coordenação de Aperfeiçoamento de Pessoal de Nível Superior (CAPES), fuente representativa para acceder a la producción académica brasileña. 
Ante los 2600 trabajos relacionados con la educación ambiental que se encuentran en el repositorio, en el momento de la recogida de datos (trabajos indexados de 1987 a 2009), solo 54 resúmenes académicos tratan dimensiones locales y/o cuestiones ambientales mundiales. De éstos, solo un tercio hace mención a ambas escalas, y en solo dos estudios se identifica la intención de discutir los posibles vínculos entre lo local y lo global. Según las autoras del estudio, esto indica claramente la falta de "fluidez en el trabajo con la articulación de estas escalas, lo que consideramos invisibiliza o dificulta la adopción de medidas eficaces para hacer frente a la actual crisis ambiental" (DIAS; BONOTTO, 2014, p.717), lo que indica la necesidad de más estudios que aborden esta cuestión.

Así, vemos que hay una brecha en cuanto a las propuestas y proyectos educativos presentados que afectan a los ámbitos locales y globales en cuanto a las cuestiones ambientales, que se refleja incluso en las investigaciones acerca de este tema, lo que nos llevó a seguir investigando sobre ello.

\section{3- Educar para una ciudadanía planetaria}

Estamos de acuerdo con Bonil, Junyet y Pujol (2010) en que es necesario un cambio en la forma de actuar de la ciudadanía en todos los aspectos de la vida para que sea posible avanzar hacia la sostenibilidad. Un cambio que es imprescindible abordar desde una perspectiva compleja, situándonos, así, en un modelo de vida que entiende la libertad desde la responsabilidad, el entendimiento político desde una democracia participativa y la comunidad como una forma de proyectarse hacia la globalidad. No siendo esto posible sino desde una educación ciudadana planetaria.

Ciudadanía e Historia de la humanidad van intrínsecamente unidas en cuanto que se desarrollan en el seno de las sociedades y de los grupos sociales. Fue en 1789, con la Revolución Francesa y el nacimiento de las llamadas nuevas sociedades democráticas, la ocasión en la cual cambió sustancialmente la forma de entender la ciudadanía, conformándose como parte integrante del Estado y no como súbdita del mismo.

Desde entonces, y de distintos contextos, el término ha adquirido diferentes significados. Para nosotros, es Tedesco (2003, p.3) quién se acerca más al cambio de concepto de ciudadanía enmarcándose dentro de las problemáticas actuales, señalando que estos cambios:

[...] tienen grandes implicaciones en la aparición de lo local y lo supranacional como nuevos espacios de participación social, asociándose a fenómenos de ruptura, de la acción política tal como se la concebía hasta ahora. La construcción de un concepto de ciudadanía mundial, de ciudadanía planetaria, exige un concepto de solidaridad vinculado a la pertenencia al género humano y no a alguna de sus formas particulares.

Para lograr una ciudadanía planetaria que sea real debemos empezar a separar el concepto de nacionalidad del de ciudadanía, dos términos que se encuentran en estrecha relación en el imaginario actual. La nacionalidad responde al ámbito (nación) al que se pertenece por vínculo de nacimiento, mientras que la ciudadanía debería ser un concepto más amplio y no limitarse únicamente al ámbito de nacimiento; debería tenerse en cuenta el ámbito donde uno se desarrolla como persona, realiza su vida o aporta a la comunidad y a las instituciones públicas... Y, por supuesto, considerar a la madre tierra como el primer y último lugar donde habitamos, donde ejercer una ciudadanía planetaria bajo valores de respeto, tolerancia, igualdad, libertad, diversidad y solidaridad.

Las diferentes acepciones del término ciudadanía planetaria pueden dar lugar a confusión, ya que son sinónimas y conllevan diferentes matices. En la terminología recurrente encontramos denominaciones como ciudadanía planetaria (BOFF, 1995; GUTIÉRREZ- 
PÉREZ, 2003), ciudadanía global (BANKS, 1997; OLU, 1997; MERRYFIELD; JARCHOW; PICKERT, 1997), ciudadanía universal (UNESCO, 1990; MEDINA, 1998), ciudadanía mundial (ONU, 1945; PASQUINO, 2001); ciudadanía cosmopolita (NUSSBAUM, 1999; CORTINA, 2003); ciudadanía ambiental o ciudadanía ecológica (CARNEIRO, 1996; VAN STEENBERGEN, 1994; DOBSON, 2001, 2005), entre otros.

Aunque el término más utilizados es el de ciudadanía global, nos hemos decantado por el uso de ciudadanía planetaria, distanciándonos del término global por su ambigüedad y considerando que el concepto de ciudadanía global "estaría mucho más ligado al reciente proceso de globalización, provocado por los avances tecnológicos; en cuanto a la planetariedad, continua siendo un deseo, un sueño que viene de mucho más lejos" (GADOTTI et al., 2003, p.100).

Asumir la planetariedad es asumir los problemas a los que se enfrenta la humanidad (hambre, pobreza, crecimiento desproporcionado, abuso en el uso de los recursos, cambio climático, lluvias ácidas, guerras, extinción de especies) como propios y ser capaces de crear un proyecto de civilizaciones establecido en base a las relaciones de la sociedad. Una concepción que, al igual que la de ciudadanía, tiene sus orígenes en la tradición griega, concretamente en la estoica, donde se formula por primera vez el término "cosmopolita, desplazando la polis (ciudad) hacia la cosmopolis (ciudad universal), y que implicaba una lealtad a la comunidad mundial frente a la comunidad en la cual uno nacía (DELANTY, 2008, p.36-37).

Una cosmopolitización que no está exenta de críticas y de dificultades para el consenso, ya que no faltan opositores que crean en la inviabilidad de esa idea de ciudadanía planetaria, argumentando factores de tipo político, económico, sociales o culturales y que sostienen que los cambios sociales resultarán en función de los conflictos sociales concretos que se produzcan en el seno de cada sociedad o nación. Factores que se reducen a una falta de voluntad por llegar a un entendimiento que dé lugar a unas bases justas para toda la ciudadanía que conforma el planeta y, sobre todo, intereses escondidos que están, en muchos casos, por encima de los derechos humanos y ambientales.

Sabemos que es utópico el pensar en una ciudadanía planetaria en el que la humanidad se conforme en un todo único, donde haya toda superación de conflictividad. La ciudadanía planetaria reconoce las diferencias, locales y globales, y apuesta por compartirlas y enriquecerse de ellas.

Coincidimos con Ferrete (2011) en la idea de que una gobernanza global permitiría ampliar los espacios y los instrumentos al servicio de la ciudadanía, fortaleciendo la conciencia y la competencia cívica, aunque sin perder de vista la educación como el factor más importante a la hora de empoderar a la ciudadanía. Una gobernanza global, complementada a escala local, regional, nacional, y global, para la que sería necesario contar con instituciones públicas globales, que estudien y aporten medidas a corto, medio y largo plazo que permitan afrontar las cuestiones relevantes que nos atañen en todos los espacios (políticos, económicos, institucionales, sociales etc.). Lo que implicaría que la ciudadanía tuviera a su disposición todos aquellos instrumentos necesarios para poder tomar decisiones y proponer iniciativas en todos aquellos ámbitos que fueran de su interés o por lo cuales le concerniera de alguna forma (GARCÍA MARZÂ, 1993; FERRETE, 2011).

En este sentido, la alternancia de estos espacios ciudadanos contribuye a la generación de una consciencia ecológica planetaria, asociada a preocupaciones que pueden alcanzar incluso la conservación el planeta, fortaleciendo valores que pueden aportar a la construcción de nuevas identidades. Se hace referencia, así, al sentimiento creciente de pertenecer a una ciudadanía planetaria, siendo estos nuevos espacios públicos escenarios privilegiados para la toma de conciencia sobre los problemas que afectan el mundo de hoy, como el adelgazamiento de la capa de ozono o el calentamiento global entre otros (DASCAL, 2007). 
En el panorama actual, esta idea de ciudadanía cosmopolita está representada entre otros autores y autoras, por Nussbaum $(1999$, p.14), firme defensora del ciudadano cosmopolita, al que define como "el comprometido con toda la comunidad de seres humanos", argumentando que la ciudadanía cosmopolita puede considerarse el núcleo de a educación cívica. Aunque esta autora concreta sobre el caso de la cultura estadounidense, pueden sus ideas extrapolarse.

Se puede afirmar que otra forma de entender las relaciones con en el planeta es posible y que está en nuestras manos hacer de él un lugar más justo, más igualitario, más de todos y más humano, sin disolución de las identidades compartidas (CASTELLS, 1998). Se hace imprescindible articular mecanismos para formar ciudadanos críticos y comprometidos, adquiriendo, así, la educación un papel fundamental en cualquier Estado, para que se produzcan cambios perceptibles y significativos.

Como ya venimos desentrañando, la educación no ha permanecido ajena a la influencia del fenómeno globalizador, los procesos educativos también han estado y están sujetos a cambios externos que han repercutido en su función social y en su funcionamiento institucional (BONAL; TARABINO-CASTELLANI; VERGER, 2007), una influencia que se ha visto reflejada en la escuela en educandos competitivos, autosuficientes, y egoístas para los que el yo está por encima del nosotros, limitándose a una visión individualista y localista que en nada favorece a las problemáticas actuales, que requieren de una visión de conjunto más que nunca.

Hecho que en el momento actual deberíamos de reconsiderar, ya que la complejidad del mundo en el que vivimos nos enfrenta a una nueva forma de vivir, de tomar conciencia, de reflexionar, de relacionarnos con los individuos, con el entorno, con el mundo.

Es ante estas nuevas realidades educativas que se están dando en las aulas, donde los educadores tienen la responsabilidad de que todos este alumnado, perteneciente a una comunidad y un contexto determinado aprenda junto, independientemente de sus características personales, sociales o culturales, dando lugar a una ciudadanía común donde las diferencias sean un valor añadido, ya que

[...] quienes se integran como ciudadanos no son ni las civilizaciones, ni las culturas, ni siquiera los grupos culturales y las identidades colectivas que representan, de estas palabras podemos deducir que son los individuos y sus singularidades quienes se integran como ciudadanos (LLAMAZARES, 2008, p.66).

Los cambios acontecidos en los últimos años, y aunque con grandes limitaciones, están transformado a la comunidad educativa en un nuevo espacio de convivencia, donde sus diferentes actores y sus códigos de normas propias, promueven la acción colectiva, potenciando planteamientos de ciudad educadora como la participación, el diálogo y el compromiso en una acción educativa crítica y transformadora (CABRERA, 2002). Es a través de la educación, que nuestros niños y niñas se socializan y adquieren una serie de hábitos, comportamientos y valores que los incorporan a una vida ciudadana plena. Como bien señala Freire (1990), las prácticas educativas son también prácticas sociales, lo que añade a estas prácticas un elemento de corresponsabilidad.

Y es en las últimas décadas, con más fuerza en los últimos años, cuando se comienza a hacer referencia a la necesidad de conformar una ciudadanía planetaria, planteándose una nueva visión de los ciudadanos. Esta ciudadanía del mundo sienta sus bases en la participación creativa de toda la comunidad, traduciéndose en la escuela en que el educador o educadora de la era planetaria tiene que vivir planetariamente, y por lo tanto educar ciudadanos con la misma filosofía.

La educación ciudadana planetaria se traduce en dar una visión de polis mundial desde la escuela, donde todos los seres humanos, independientemente del lugar del mundo donde se encuentren, se sientan parte de un mismo proyecto de vida, y con la misma finalidad: la de 
participar, de forma activa y responsable, en las decisiones que afectan a nuestro hogar, nuestro planeta. Siendo algunos de sus objetivos principales (MORENO, 2011):

- Sensibilizar al alumnado de las problemáticas sociales, ambientales y económicas en las que se encuentra la situación del planeta.

- Promover la participación social del alumnado desde su inclusión en la escuela.

- Promover el cumplimiento de las normas como base de una ciudadanía democrática y crítica.

- Favorecer el conocimiento personal y el aprecio por las diferentes formas culturales viéndolas como enriquecimiento personal y social.

- Fomentar el ejercicio de valores democráticos a través de situaciones que se dan en la vida cotidiana.

- Enseñar a pensar de forma reflexiva, crítica, integral y planetaria.

- Fomentar una cultura de colaboración y no violencia, promotora de los valores radicales de la dignidad humana, que fomente la protección ambiental, la justicia social y económica, y el respeto a la diversidad y la integridad cultural y ecológica (NOVO; MURGA, 2010, p.5).

- Aportar conocimientos sobre los elementos, factores y agentes económicos, sociales y políticos que explican la dinámica de la sociedad en que vivimos y provocan la existencia de pobreza, marginación, desigualdad y opresión condicionando la vida de las personas (BENITO-MARTÍNEZ, 2006, p.8).

Pero para que estos objetivos sean una realidad en la práctica escolar, es necesario que los docentes estén plenamente preparados para asumir la planetariedad a la que venimos refiriéndonos. Y esto no es posible más que asumiendo los problemas en los que nos encontramos inmersos y asumiendo un proyecto de civilizaciones que se asiente en las relaciones de las sociedades (GUTIÉRREZ-PEREZ, 2003), donde los educandos participen activamente a través de la reflexión crítica y la creatividad.

La ciudadanía planetaria es una forma de entender las relaciones con los otros, entendiendo por otros a todo aquello que está fuera de uno mismo. Es una sinergia entre el yo y el mundo, donde cada acción local conlleva, inexorablemente, una reacción global, y donde el ser humano tiene su mayor responsabilidad, la de la preservación del medio, pues "procedemos de la Tierra, somos de la Tierra, estamos en la Tierra. Pertenecemos a la Tierra que nos pertenece" (MORIN; KERN, 2005, p.221).

Estas problemáticas a las que nos enfrentamos actualmente, y que abarcan a todos los ámbitos en los que nos desenvolvemos, exigen que se den respuestas tanto a nivel nacional como internacional, tanto a nivel local como global. Es necesaria una respuesta mundial unificada y consensuada, que de soluciones efectivas más allá de simples aportaciones que se quedan en propuestas sin salidas. Concordamos con Gadotti (2000, p.8) que tal educación implica mucho más que una filosofía educativa, "significa una revisión de nuestros currículos, una reorientación de nuestra visión del mundo de la educación como espacio de inserción del individuo no en una comunidad local, sino en una comunidad que es local y global al mismo tiempo".

\section{4- Consideraciones finales}

A partir del cuadro general presentado podemos reconocer que la realización por parte de la escuela de actividades educativas dirigidas para una ciudadanía orientada a hacer frente a los actuales problemas socio-ambientales no constituye una tarea simple. La interrelación localglobal, si no se trata adecuadamente, puede implicar en un reduccionismo que debilita los resultados educacionales que estamos intentando lograr en términos de la educación para una ciudadanía planetaria. 
Para nosotros ese es el objetivo que debemos plantear, aprovechando todo lo que la Educación Ambiental y demás corrientes de educación orientadas a la ciudadanía han aportado a la comprensión de la actual situación planetaria. Como destaca García Diaz (2006, p.515), "debemos hacerlo conscientes de las dificultades, pero decididos a contribuir, como educadores, como científicos y como ciudadanos, a forjar las condiciones de un fututo sostenible".

Para cerrar este ensayo, además de otras cuestiones que se han presentado, seguimos incidiendo en una cuestión que consideramos como fundamental, la necesidad de cuidar la formación de los educadores. Mientras buscamos un profundo cambio de visión del mundo en el que vivimos, nos acercamos con las consideraciones de Morin (1999), para quien la superación de la actual situación agónica planetaria implica una profunda reforma del pensamiento, que, según él, sólo puede llevarse a cabo a través de la reforma de la educación. Es más, es el mismo autor quien plantea, apoyándose en Marx: ¿quién educará a los educadores? Es preciso que ellos se eduquen a sí mismo (MORIN, 1999, p.34).

Aunque esta afirmación pueda parecer demasiado simplista, al parecer, sugiriendo aparentemente que los maestros estarían dejados a su suerte, nos permite entrar en el tema que completa el foco de interés de este artículo, presentando una cuestión que emerge de las reflexiones anteriormente expuestas: dada la complejidad de la propuesta para trabajar con conexiones locales-globales para educar hacia una ciudadanía planetaria, sólo podemos reflexionar sobre la figura del maestro, sin el cual no hay cambio educativo que se lleva a cabo.

Hay que se ofrecer propuestas de formación de docentes centradas en estas discusiones, con el fin de equipar a los educadores para elaborar propuestas educativas y tratar apropiadamente de este tema con sus alumnos. La literatura consultada, así como las investigaciones llevadas a cabo por nosotros, nos han demostrado que este tema no ha sido priorizado en los cursos de formación para profesores. Corresponde a la universidad colaborar con este proceso, presentando propuestas de formación que aborden esta cuestión, así como la inversión en investigaciones en este sentido, indicando sus límites y posibilidades.

Concordando, una vez más, con las directrices presentadas en el Tratado de Educación Ambiental para Sociedades Sostenibles y Responsabilidad Global con relación a una educación ambiental, frente a este reto, todos somos aprendices, todos tenemos mucho que aprender por delante.

\section{Referencias}

ABDEL-MONEIM, S. G. O Ciborgue Zapatista: tecendo a poética virtual de resistência no Chiapas cibernético. Estudos feministas, Florianópolis, v.7, n.1-2, p.39-64, Ene./Jun. 2002.

BANKS, J. A. Educating Citizens in a Multicultural Society. New York: Teachers Columbia University, 1997.

BENITO-MARTÍNEZ, J. Educación y ciudadanía. Eikasia - Revista de filosofía, Oviedo, Año II, n. 6, p.1-17, sept. 2006.

BOFF, L. Nueva era - la civilización planetaria: desafíos a la sociedad y al cristianismo. Navarra: Verbo Divino, 1995.

BONAL, X.; TARABINO-CASTELlANI, A. VERGER, A. (Comp.). Globalización y educación. Textos fundamentales. Buenos Aires: Miño y Dávila, 2007. 
BONIL, J; JUNYENT, M; PUJOL, R.M. Desarrollo de una cartografía de investigación en el paradigma de la complexidad para avanzar en la educación para la sostenibilidad. CONGRESO INTERNACIONAL DE DIDÁCTICAS, 2, Girona, 2010. Girona: Departamento de didácticas específicas de la Universidad de Girona, 2010. p.148-155. Disponível em: < http://www.researchgate.net/publication/44789079_Desarrollo_de_una_cartografa_de_investi gacin_en_el_paradigma_de_la_complejidad_para_avanzar_en_la_educacin_para_la_sostenibi lidad>. Acesso em: 15 jun. 2015.

CABRERA, F. Hacia una nueva concepción de la ciudadanía en una sociedad multicultural. En BARTOLOMÉ, M. (Coord.). Identidad y Ciudadanía. Un reto a la educación intercultural. Madrid: Narcea, 2002. p. 79-204.

CARNEIRO, R. La relativización de la educación y las comunidades humanas: una visión de la escuela socializadora del siglo XXI. En UNESCO (Ed.). La educación encierra un tesoro. Informe UNESCO de la Comisión Internacional sobre educación para el siglo XXI. Madrid: Santillana/UNESCO, 1996. p. 241-246.

CASTELLS, M. La era de la información. Economía, sociedad y cultura. Madrid: Alianza, 1998.

CORTINA, A. Hacia un concepto de ciudadanía para el siglo XXI. Misión Joven, $\mathrm{N}^{\mathrm{o}}$ 314, pp.17-24, 2003. [Vol. 3].

DASCAL, G. Los espacios públicos y el capital social: aportes para comprender la relación entre ambos conceptos. Cuadernos de geografía, Bogotá, v. 1, n. 16, p.19-26, Ene. /Dic. 2007.

DIAS, G. M.; BONOTTO, D. M. B. As dimensões local e global nos entendimentos e práticas de professores participantes de um curso de formação continuada em educação ambiental. Revista Electrónica de Enseñanza de las Ciencias, Vigo, v.11, n.1, p.145-163, En./Abr. 2012.

DIAS, G. M.; BONOTTO, D. M. B. As escalas local e global apresentadas em teses e dissertações brasileiras de educação ambiental. Ciênc. Educ., Bauru, v.20, n.3, p.703-719, Jul./Set. 2014.

DELANTY, G. La imaginación cosmopolita. Revista CIDOB d'Afers Internacionals, Barcelona, v.2, n.82-83, p.35-49, Sept. 2008.

DOBSON, A. Ciudadanía ecológica: ¿una influencia desestabilizadora?, Isegoría Revista de filosofía, moral y política, Madrid, v.0, n.24, p.167-187, Jun. 2001.

DOBSON, A. Ciudadanía ecológica, Isegoría, Revista de filosofía, moral y política, Madrid, v.0, n.32, p.47-62, Jun. 2005.

FERRETE, C. Ciudadanía sin límites: el trasfondo de la gobernanza global. Quaderns de filosofia i ciència, Valencia, v. 1, n.41, p.89-98, Ene./Dic. 2011.

FREIRE, P. La naturaleza política de la educación. Barcelona: Paidós, 1990.

GADOTTI, M. Pedagogía de la Tierra. Sao Paulo: Fundação Petrópolis, 2000. 
GADOTTI, M. et al. Perspectivas actuales de la educación. México: Siglo XXI, 2003.

GARCÍA DÍAZ, J. E. Educación ambiental y alfabetización científica: argumentos para el debate. Investigación en la Escuela, Sevilla, v.3, n.60, p.7-19, Dic. 2006.

GARCÍA MARZÁ, D. Teoría de la democracia. Valencia: Nau Llibres, 1993.

GASKELL, J.; OGAWA, M. Globalization and Localization: competing tendencies or inevitable companions? En: INTERNATIONAL ORGANIZATION FOR SCIENCE AND TECHNOLOGY EDUCATION- IOSTE, 11, Lublin, 2004. Proceedings ... Lublin: Maria Curie - Sklodowska University Press, 2004. p. 29-30.

GIDDENS, A. "Admirável mundo novo: o novo contexto da política". Cad. $C R H$, Salvador, v.7, n.21, p.9-28, 1994. Disponible en: <http://www.cadernocrh.ufba.br> Acceso: 28 abr. 2011.

GUIMARÃES, M. Educação Ambiental: participação para além dos muros da escola. En MELLO, S. S. de; TRAJBER, R. (Coords). Vamos cuidar do Brasil: conceitos e práticas em educação ambiental na escola. Brasília: Ministério da Educação, Coordenação Geral de Educação Ambiental y Ministério do Meio Ambiente, Departamento de Educação Ambiental/UNESCO, 2007. p.85-94.

GUTIERRÉZ-PÉREZ, F. Ciudadanía planetaria. En MARTÍNEZ BONAFÉ, J. (Coord.). Ciudadanía, poder y educación. Barcelona: Graó, 2003. p. 133-155.

LAYRARGUES, P. P. A resolução de problemas ambientais locais deve ser um tema-gerador ou a atividade-fim da educação Ambiental? En REIGOTA, M. (Coord.). Verde cotidiano: meio ambiente em discussão. Rio de Janeiro: DP \& A, 1999. p. 131-148.

LLAMAZARES, D. Educación para la ciudadanía democrática y objeción de conciencia. Madrid: Dykinson, 2008.

LOUREIRO, C.F. B. Teoria Social e questão ambiental: pressupostos para uma práxis crítica em educação ambiental. In: LOUREIRO, C.F.B; LAYRARGUES, P.P.; CASTRO, R.S. (Orgs.) Sociedade e meio ambiente: a educação ambiental em debate. São Paulo, Cortez Editora, 2000, p. 13-51.

MAYER, M. Ciudadanos del barrio y del planeta. En IMBERNÓN, F. (Coord). Cinco ciudadanías para una nueva educación. Barcelona: Graó, 2002. p. 83-104.

MERRYFIELD, M. M; JARCHOW, E.; PICKERT, S. (Eds.). Preparing teachers to teach global perspectives: A handbook for teacher educators. California: Corwin Press, 1997.

MORENO, O. La escuela como base para una ciudadanía planetaria. Inclusividad y participación ciudadana. Revista Quaders Digitals, Valencia, v. 4, n.69, p.1-15, oct. 2011.

MORENO, O. Educación ambiental y educación para la ciudadanía desde una perspectiva planetaria. Estudio de experiencias educativas en Andalucía. 2013. 694 pág. Tesis (Doctorado en Estudios Medioambientales) - Universidad Pablo de Olavide, Sevilla, 2013. 
MORIN, E. Por uma reforma do pensamento. In: PENA-VEGA, A. e NASCIMENTO, E.P. (Orgs.) O pensar complexo: Edgar Morin e a crise da modernidade. Rio de Janeiro: Garamond, 1999, p. 21-34.

MORIN, E. ; KERN, A.B. Tierra-patria. Barcelona: Editorial Kairós, 2005.

NOVO, M.; MURGA, M. de los A. Educación ambiental y ciudadanía planetaria. Revista Eureka sobre enseñanza y divulgación de las ciencias, Cádiz. v.7, n. Extraordinario, p.179-186, Feb. 2010.

ONU. Organización de Naciones Unidas. Carta de las Naciones Unidas. Firmada el 26 de junio 1945 entrada en vigor: 24 de octubre de 1945, de conformidad con el artículo 110. San Francisco/EUA: Organización de Naciones Unidas, 1945.

NUSSBAUM, M. Patriotismo y cosmopolitismo. En: NUSSBAUM, M. y COHEN, J. (Ed.). Los Límites del Patriotismo. Identidad, Pertenencia y «Ciudadanía Mundial». Barcelona: Paidós, 1999. p. 13-29.

OLU, S. Models of multiculturalism: implications for the twenty-firt century leaders. European Journal of Intercultural Studies, USA, v.3, n.8, p.231-256, Jul.1997.

PASQUINO, G. Ciudadanía mundial. Psicología Política, Valencia, v.2, n.23, p.59-75, Nov. 2001.

PORTO-GONÇALVES, C.W. Educação, meio ambiente e globalização. En: CONGRESSO IBERO-AMERICANO DE EDUCAÇÃO AMBIENTAL, 5, Joinville, 2006. Anais... Rio de Janeiro: Associação Projeto Roda Viva, 2007. p.15-27.

RODRIGUES, N. Educação: da formação humana à construção do sujeito ético. Educação \& Sociedade, Campinas, v.22, n.76, p. 232-257, Oct. 2001.

SANTOS, M. Por uma outra globalização: do pensamento único à consciência universal. São Paulo: Record, 2000.

SANTOS, M. Por uma outra globalização: do pensamento único a consciência universal. Rio de Janeiro: Record, 2002.

SAUVÉ, L. Environmental Education and Sustainable Development: a Further Appraisal. Canadian Journal of Environmental Education, Ontario, v.1, n.1, pp.7-34, 1996.

TEDESCO, J. C. Los pilares de la educación del futuro. En: Debates de educación [ponencia en línea]. Barcelona: Fundación Jaume Bofill/UOC, 2003. s/p. Disponible en: <http://www.uoc.edu/dt/20367/index.html >. Acceso: 18 abr. 2014.

VAN STEENBERGEN, B. Towards a Global Ecological Citizen. En: VAN STEENBERGEN, B. (Ed.). The Condition of Citizenship. London: Sage, 1994. p. 141-152.

VIEZZER, M.; OVALLES, O. Manual Latino-americano de Educação ambiental. São Paulo: Editora Gaia, 1994. 
VILARRASA, A. El medio local como escala de análisis en la didáctica de las Ciencias Sociales de Educación Secundaria. Enseñanza de las Ciencias Sociales, Barcelona, v.4, n.4, p.11-19, Feb. 2005.

WILBANKS, T. J.; KATES, R.W. Global change in local place: how scale matters. Climatic Change, Princenton, v.43, n.3, p. 601-628, Nov. 1999. 